\title{
Baufieber und Stiftungseifer Überlegungen zum Einfluss der Pfarrgemeinde auf die Gestaltung des sakralen Raumes: das Beispiel Zwickau
}

\author{
von \\ JULIA KAHLEYSS
}

Im Jahr 1536, als die jahrzehntelangen Arbeiten an der größten Zwickauer Stadtkirche, St. Marien, mit der Fertigstellung des Kirchendaches zu einem vorläufigen Ende kamen, beschloss der Zwickauer Rat, eine Erinnerungstafel aus Messing am Gewölbe anzubringen. Die Inschrift darauf sollte neben dem kurfürstlichen Wappen auch das größte Ratssiegel beinhalten und vermerken, wenn und bey webme dieser baw sey volendet worden. ${ }^{1}$

Der Zwickauer Rat hatte auch allen Grund, seinem Stolz über die Vollendung des Bauwerkes Ausdruck zu verleihen, denn damit war aus einer Kirche, die Mitte des 15. Jahrhunderts einen schmalen und wohl recht baufälligen Chor und ein ungleich größeres gotisches Langhaus besaß, eine der größten und beeindruckendsten Hallenkirchen des Erzgebirges geworden. ${ }^{2}$

Bereits Hartmut Boockmann wies in seiner 1992 gehaltenen Antrittsvorlesung über „Bürgerkirchen im späteren Mittelalter“ auf die Signalfunktion der Größe und der reichen Ausstattung der spätmittelalterlichen Kirchenbauten hin. ${ }^{3}$ Ein Bündel von verschiedenen, kaum trennbaren Motiven, die zwischen „Devotion und Repräsentationswünschen" schwankten, brachte die beteiligte Geistlichkeit, den Adel und die städtischen und dörflichen Gemeinden dazu, die Kirchen so groß wie möglich zu bauen und mit kostbaren Kunstwerken auszustatten. ${ }^{4}$ Die Zwickauer Pfarrkirche St. Marien mit ihrer reichen Überlieferung eignet sich sehr gut, um den Versuch zu unternehmen, einige der vielen Bestandteile dieses „Motivbündels" an ihrem konkreten Beispiel zu analysieren.

1 Stadtarchiv Zwickau (im Folgenden: StA Zwickau), IIIx, 64/159, 60b.

2 Michael Kirsten, Der Dom St. Marien zu Zwickau (Große Kunstführer 200), Regensburg 1998, S. 5. Der vorliegende Aufsatz ist eine Weiterentwicklung von Abschnitten aus: Julia KaHLEYss, Die Bürger von Zwickau und ihre Kirche. Kirchliche Institutionen und städtische Frömmigkeit im späten Mittelalter (Schriften zur sächsischen Geschichte und Volkskunde 45), Leipzig 2013.

3 Hartmut Boockmann, Bürgerkirchen im späteren Mittelalter. Antrittsvorlesung 3. November 1992 (Öffentliche Vorlesungen der Humboldt-Universität zu Berlin 30), Berlin 1994, S. 13.

4 Ebd., S. 15; vgl. dazu auch Martin Warnke, Warum sind mittelalterliche Kirchen so groß?, in: Norbert Nußbaum (Hg.), Die gebrauchte Kirche. Symposium und Vortragsreihe anlässlich des Jubiläums der Hochaltarweihe der Stadtkirche Unserer Lieben Frau in Friedberg (Hessen) 1306-2006 (Arbeitshefte des Landesamtes für Denkmalpflege Hessen 15), Stuttgart 2010, S. 162-168. 
Die Marienkirche blickt seit ihren Anfängen, die mit dem ersten Kirchenbau in Form einer romanischen Saalkirche wohl um das Jahr 1180 anzusetzen sind, auf eine wechselvolle Baugeschichte zurück. ${ }^{5}$ Im Laufe der Jahrhunderte war die Kirche mehrfach von Stadtbränden betroffen, die einige An- und Umbauten erforderlich machten. ${ }^{6}$ Um die Mitte des 15. Jahrhunderts beförderten vermutlich Bauschäden an Turm und Seitenhallen und die Diskrepanz zwischen dem breiten gotischen Langhaus und dem schmalen romanischen Chor die Entscheidung zum Abriss des alten und zum Bau eines neuen, breiteren Chores, ${ }^{7}$ dessen Gestaltung sich wahrscheinlich an fränkische Vorbilder, vor allem an den Chor der St. Lorenzkirche in Nürnberg, anlehnt. ${ }^{8}$ Von 1453 bis 1470,9 nach anderen Angaben bis

5 Zur Kirchengeschichte Zwickaus seit dem 12. Jahrhundert vgl. Leo Bönhoff, Die Gauparochie Zwickau, in: Beiträge zur sächsischen Kirchengeschichte 32 (1919), S. 6499; Ders., Der Gau Zwickau, in: NASG 40 (1919), S. 241-295; Walter Schlesinger, Die Anfänge der Stadt Chemnitz und anderer mitteldeutscher Städte. Untersuchungen über Königtum und Städte während des 12. Jahrhunderts, Weimar 1952, S. 151, 171; Manfred Ковuсн, Zur Frühgeschichte Zwickaus. Bemerkungen zu Stadt und Vorstadt im 12. und 13. Jahrhundert, in: Regionalgeschichtliche Beiträge aus dem Bezirk Karl-Marx-Stadt 2 (1980), S. 49-64. Oelsner, Stoye und Walther datieren die Entstehungszeit der Marienkirche aufgrund neuerer Ausgrabungsergebnisse genauer auf das letzte Viertel bzw. Ende der achtziger Jahre des 12. Jahrhunderts, vgl. Norbert OelsNer/Wilfried Stoye/Thomas Walther, Marienkirche und Nikolaikirche in Zwickau. Neue Erkenntnisse zur Frühgeschichte der Stadt, in: Judith Oexle (Hg.), Frühe Kirchen in Sachsen. Ergebnisse archäologischer und baugeschichtlicher Untersuchungen (Veröffentlichungen des Landesamtes für Archäologie mit Landesmuseum für Vorgeschichte 23), Stuttgart 1994, S. 161-165; Norbert Oelsner, Die Marienkirche in Zwickau. Erkenntnisse zu ihrer mittelalterlichen Baugeschichte und zur Entstehung der Stadt, in: Denkmalpflege in Sachsen 1894-1994, 2. Teil, hrsg. vom Landesamt für Denkmalpflege Sachsen, Halle a. d. Saale 1998, S. 209-232, was Beutmann kritisch als nicht ausreichend belegt anzweifelt und auf einen Entstehungszeitraum in der zweiten Hälfte des 12. Jahrhunderts verweist, vgl. Jens Beutmann, Untersuchungen zu Topographie und Sachkultur des mittelalterlichen Zwickau. Die Ausgrabungen im Nordwesten des Stadtkerns (Veröffentlichungen des Landesamtes für Archäologie mit Landesmuseum für Vorgeschichte 49), Dresden 2007, S. 197. Michael Kühn verweist dagegen auf die Möglichkeit, dass der erste Kirchenbau der Marienkirche nicht im Dorf Osterwein, sondern an der Stelle der heutigen Marienkirche in den Altarraum integriert worden sein könnte, vgl. Michael KüHN, Baugeschichte der St. Marienkirche Zwickau, seit 1935 „Dom St. Marien Zwickau“, in: Informationshefte Dom St. Marien 14 (2010), S. 4-16, hier S. 5.

6 Kirsten, Dom St. Marien (wie Anm. 2), S. 6-8; Kühn, Baugeschichte (wie Anm. 5), S. 8.

7 Karl Weissbach, Die Marienkirche in Zwickau. Ein Beitrag zur Kenntnis ihrer Baugeschichte und ihrer Beziehungen zum Erzgebirgischen Kirchenbau, Zwickau 1922, S. 12 .

8 Helga Baier, Der Dom St. Marien zu Zwickau (Das christliche Denkmal 104), Berlin 1989, S. 5; Dietlinde Brugmann, Art. Zwickau, in: Georg Dehio, Handbuch der Deutschen Kunstdenkmäler, Sachsen II: Regierungsbezirke Leipzig und Chemnitz, bearb. von Barbara Bechter u. a., München 1998, S. 1076-1103, hier S. 1082; Susan C. Karant-Nunn, Zwickau in Transition: 1500-1547. The Reformation as an Agent of Change, Columbus 1987, S. 17.

9 Weissbach, Marienkirche (wie Anm. 7), S. 13. 
1475,10 dauerten die Bauarbeiten an. Bereits 1476 wurden die Bauarbeiten fortgesetzt, wohl aufgrund von Schäden am Gebäude, die von Statikproblemen des Kirchturmes verursacht wurden. ${ }^{11} \mathrm{Um}$ den Turm zu stützen, wurden nach einem Plan des Baumeisters Arnold von Westfalen und unter der Leitung des Zwickauer Baumeisters Nickel Eichhorn die beiden nord- und südöstlich gelagerten Vorhallen gebaut. ${ }^{12}$

Die nächste große Bauphase kündigte sich bereits Ende des 15. Jahrhunderts an. Neben weiteren Baumängeln am gotischen Langhaus hatte sich jenes auch als zu klein für den Platzbedarf der schnell wachsenden Stadt Zwickau erwiesen. ${ }^{13}$ Die Entscheidung über die Vorgehensweise bei der Sanierung des Langhauses stand mehrere Jahre lang auf der Agenda des Zwickauer Rates. Einige auswärtige Baumeister wurden um Gutachten gebeten, beispielsweise 1495 der Leipziger Werkmeister Claus Roder, ${ }^{14}$ Meister Hans, des Herzogen parlirer, ${ }^{15}$ oder 1497 Meister Symon, ${ }^{16}$ der vermutlich zu dieser Zeit den Bau des Langhauses des Freiberger Domes leitete. Schließlich entschied sich der Rat für einen Neubau des Kirchenschiffes und beauftragte damit den Baumeister Peter Heierliß/Harlass aus Jena. Heierliß starb jedoch bereits 1507, sodass sein Plan, das Langhaus zu verbreitern und damit einen harmonischen Übergang vom Langhaus zum Chor zu erreichen, erst von seinem Nachfolger Caspar Teicher realisiert werden konnte. ${ }^{17}$

Im Laufe der jahrzehntelangen Bauzeit traten Schwierigkeiten mit dem Dach des Hallenbaus und finanzielle Probleme auf, die kurzzeitig die Unterbrechung der Bauarbeiten erzwangen. Vom April 1535 bis Juni 1536 folgte schließlich als letzter Schritt vor der Fertigstellung die Einwölbung des Langhauses. ${ }^{18}$ Der Stadt-

10 KüHN, Baugeschichte (wie Anm. 5), S. 8.

11 Ebd., S. 9; Weissbach, Marienkirche (wie Anm. 7), S. 16.

12 Kirsten, Dom St. Marien (wie Anm. 2), S. 9; zur Familie Eichhorn vgl. Weissbach, Marienkirche (wie Anm. 7), S. 15. Nickel Eichhorn stammte aus einer Steinmetzfamilie. Sein Vater Hans Eichhorn erwarb 1463 das Bürgerrecht. Er hatte drei Söhne, von denen zwei, Michel und Nickel Eichhorn, ebenfalls Steinmetze wurden. Michel Eichhorn arbeitete ab 1480 an der Katharinenkirche als Baumeister. Nickel Eichhorn arbeitete noch bis 1504 an der Marienkirche.

13 Kirsten, Dom St. Marien (wie Anm. 2), S. 10.

14 Ebd., S. 11; Heinrich Magirius, Der Dom zu Freiberg (Große Kunstführer 184), Regensburg 1993, S. 20; vgl. auch StA Zwickau, III Z ${ }^{4 k}$, Nr. 70, Rechnungen Marienkirche, Bd. 2: 1490-1500, Nr. 4 (1494-1495), fol. 19r: IX g einem poten gen Leipzigk der hat den werckmeister $z u$ sannd Thomas zu besichtigung vnnsers gepewdes herauffgeholt in der wochen Donati.

15 StA Zwickau, III Z4k, Nr. 70, Rechnungen Marienkirche, Bd. 2: 1490-1500, Nr. 5 (1495-1496), fol. 14r: XL g gegebenn meyster Hansen des berczogen parlirer zcw eynem geschenck das er hat den paw an der kirchen besichtiget unde seynen rath darzcw gegeben gescheen am sunabent nach Mauricy ym 95.

16 StA Zwickau, III Z ${ }^{4 k}$, Nr. 70, Rechnungen Marienkirche, Bd. 2: 1490-1500, Nr. 6 (1496-1497), fol. 15r: 4 fl. rh gegeben maister Symon dem parlirer von Freyberg, das er hat den paw besichtiget hat an der kirchen, am donerstag nach pfingsten 97 jarr, 18. Mai 1497.

17 Weissbach, Marienkirche (wie Anm. 7), S. 22 f.

18 KüHN, Baugeschichte (wie Anm. 5), S. 11. 
Julia Kahleyß

rat holte sich Rat bei Meister Conrad von Torgau und Paul Speck. ${ }^{19}$ Auf Wunsch des Stadtrates reiste Caspar Teicher nach Schneeberg, um das neu gebaute und erst 1526 fertiggestellte Dach von St. Wolfgang, einer dreischiffigen Hallenkirche mit einem 36 Meter hohen Satteldach, ${ }^{20} \mathrm{zu}$ studieren, ${ }^{21}$ sodass anzunehmen ist, dass sich Teicher bei der Einwölbung des Langhauses der Marienkirche an mitteldeutschen Vorbildern, vor allem am Bauplan der Wolfgangskirche orientierte.

\section{Die politischen Rabmenbedingungen: der Erwerb des Nominationsrechtes 1504/05}

Wie aus dem eingangs zitierten Beschluss des Rates zur Anbringung einer Gedenktafel 1536 hervorgeht, verstand sich der Zwickauer Rat als Bauherr des Neubaus der Marienkirche, was an dieser Stelle zum Anlass genommen werden soll, um einige Überlegungen über die Rolle des Rates und die Bedeutung des Bauvorhabens für die Stadt anzustellen. Klaus Jan Philipp ging in seiner Arbeit über die Pfarrkirchen der schwäbischen Reichsstädte der Frage nach, ob und inwieweit sich an der Verantwortungsübernahme und der Finanzierung eines aufwendigen Kirchenbaus durch einen Stadtrat Anstrengungen zum Gewinn von niederen Pfründen oder des Pfarrerwahlrechts nachvollziehen lassen. ${ }^{22}$ Auch die aktuelle Forschung widmet sich diesem Thema, was sich beispielsweise an dem Beitrag von Marc Carel Schurr auf der Tagung des Konstanzer Arbeitskreises für mittelalterliche Geschichte im Frühjahr 2009 zeigte. ${ }^{23}$

Philipp ging davon aus, dass insbesondere der kostspielige, von der Stadt finanzierte Ausbau eines Chores, dessen Baulast normalerweise bei dem jeweiligen Patronatsherrn lag, einen Affront gegenüber diesem und ein Zeichen für die ge-

19 Ebd.

20 Heinrich Magirius, Schneeberg. St. Wolfgang, Landshut 1995, S. 11.

21 Kirsten, Dom St. Marien (wie Anm. 2), S. 12.

22 Klaus Jan Philipp, Pfarrkirchen. Funktion, Motivation, Architektur. Eine Studie am Beispiel der Pfarrkirchen der schwäbischen Reichsstädte im Spätmittelalter (Studien zur Kunst- und Kulturgeschichte 4), Marburg 1987, S. 142.

23 Marc Carel Schurr, Architektur als politisches Argument. Die Pfarrkirche als Bauaufgabe der mittelalterlichen Städte im Südwesten des Reiches, in: Enno Bünz/Gerhard Fouquet (Hg.), Die Pfarrei im späten Mittelalter (Konstanzer Arbeitskreis für Mittelalterliche Geschichte, Vorträge und Forschungen 77), Ostfildern 2013, S. 259-278. In ähnlicher Weise kam auch Claudia Wels in ihrer 2003 eingereichten Dissertation über die Pfarrkirche St. Valentinus und die spätgotischen Landkirchen im Rheingau (http:// Archiv.Ub.uni-marburg.de/diss/z2007/0152) zu dem Ergebnis, dass sich an den Neuund Umbauten der untersuchten Kirche, der „Machtanspruch, den die Gemeinde beziehungsweise die Kirchengemeinde in Kiedrich auf ihre Pfarrkirche erhob“, dokumentiere, vgl. Claudia Wels, Die Pfarrkirche St. Valentinus in Kiedrich und ihr „städtischer" Charakter, in: Stephanie Hahn/Michael H. Sprenger (Hg.), Herrschaft Architektur - Raum. Festschrift für Ulrich Schütte zum 60. Geburtstag (Schriften zur Residenzkultur 4), Berlin 2008, S. 17-31, hier S. 29. 
plante Übernahme des Patronatsrechtes darstellte. ${ }^{24}$ Hohe finanzielle Aufwendungen für einen Neu- oder Umbau verschafften dem Rat die „Legitimation“ für die Beanspruchung der Patronatsrechte. ${ }^{25}$ Überprüft man die These anhand der Geschichte der Marienkirche, dann lassen sich im 15. Jahrhundert tatsächlich Bemühungen vonseiten des Zwickauer Rates feststellen, das Patronatsrecht über die Zwickauer Kirchen, die seit 1212 dem Nonnenkloster Eisenberg gehörten und später auch inkorporiert wurden, ${ }^{26} \mathrm{zu}$ erwerben. Obwohl es ihm erst 1504 gelang, dem Kloster zumindest das Nominationsrecht abzukaufen, ${ }^{27}$ belegt ein im Stadtarchiv Zwickau erhaltener Schriftwechsel, der die Verhandlungen beim Kauf des Nominationsrechtes von 1499 bis 1506 näher beleuchtet, dass die Zwickauer mindestens seit 1499 Anstrengungen zur Übernahme des Patronatsrechtes unternommen hatten. ${ }^{28}$ In dem ersten darin erhaltenen Schreiben aus dem Jahr 1499 berichtet Hans Monch, der Amtmann von Jena, dass er in Amtsgeschäften verhindert sei und deswegen einen geplanten Termin über Verhandlungen in Eisenberg über die Übernahme des Patronatsrechtes verschieben musste. ${ }^{29}$ Der ernestinische Kurienprokurator Dr. Ulrich Sack, den der Zwickauer Rat mit Verhandlungen in Rom über die Übernahme des Patronatsrechtes beauftragt hatte, schrieb 1505, dass sich der Zwickauer Rat oftmals bemubet [habe] auf unser liben frawen kirchen albir zw Zwickaw welche den closter junckfrawen zw Isenburg eingeleibt und incorporirt ist ius nominandi et patronatus zw erlangen. ${ }^{30}$ Folgt man Philipps These, dass die Finanzierung eines Chorneu- und -umbaus als Signal für städtische Ambitionen auf den Erwerb des Patronatsrechtes gedeutet werden kann, dann reichten die Bestrebungen des Zwickauer Rates mindestens bis zur Mitte des 15. Jahrhunderts

24 Philipp, Pfarrkirchen (wie Anm. 22), S. 142; vgl. auch Schurr, Architektur (wie Anm. 23), S. 261. Auch in Nürnberg, der Stadt, die im Allgemeinen als, Vorbild' Zwickaus gilt, lassen sich bereits im 14. Jahrhundert ähnliche Entwicklungen festhalten. Der Chorneubau der Sebalduskirche in Nürnberg stand in unmittelbarem Zusammenhang mit Unabhängigkeitsbestrebungen der Nürnberger von der Mutterpfarrei Poppenreuth, vgl. Gerhard Weilandt, Die Sebalduskirche in Nürnberg. Bild und Gesellschaft im Zeitalter der Gotik und Renaissance (Studien zur internationalen Architektur und Kunstgeschichte 47), Petersberg 2007, S. 72.

25 SchurR, Architektur (wie Anm. 23), S. 264.

26 Vgl. die Edition der Urkunde des Naumburger Bischofs Engelhard vom 14. Mai 1212, die die Übertragung der Zwickauer Kirchen an das durch Markgraf Dietrich von Meißen nach Zwickau versetzte Nonnenkloster Triptis bestätigte, vgl. Codex diplomaticus Saxoniae regiae (im Folgenden: CDS) I/3: Urkunden der Markgrafen von Meißen und Landgrafen von Thüringen 1196-1234, hrsg. von Otto Posse, Leipzig 1898, S. 129 f., Nr. 166; Hans Patze/Josef Dolle (Hg.), Urkundenbuch des Hochstifts Naumburg, Bd. II: 1207-1304 (Quellen und Forschungen zur Geschichte SachsenAnhalts 2), Weimar 2000, S. 9 f., Nr. 8.

27 Der Vertrag selbst hat sich leider nicht erhalten, vgl. Thüringisches Hauptstaatsarchiv Weimar (im Folgenden: ThHStA Weimar), EGA Reg. Oo, Findbuch, fol. 151v: Vertrag zwischen dem Closter und dem Rath zu Zwigkau belangend das Pfarrlebn daselbst anno 1504.

28 StA Zwickau, A*A II 18, Nr. 38.

29 StA Zwickau, A*A II 18, Nr. 38 (1).

30 StA Zwickau, A*A II 18, Nr. 38 (5). 
zurück, als der Chor der Marienkirche ab 1453 neu erbaut wurde. Zwar sind aus dieser Zeit keine Kirchenrechnungen erhalten geblieben, die dies belegen könnten, aber der Zwickauer Chronist Peter Schumann berichtet ausdrücklich, dass die Grundsteinlegung im Juli 1453 während der Amtszeit des Bürgermeisters Nikolaus Sturm und der beiden Kirchenmeister Matthes Richter und Johann Rott erfolgt sei, ${ }^{31}$ was in einer über der kleinen Altarpforte auf der Südseite des Chores angebrachten Inschrift Bestätigung findet. ${ }^{32}$ Die Mitwirkung des Klosters Eisenberg bei der Finanzierung des Chorneubaus wird dagegen in keiner, wenn auch städtischen und daher ,befangenen', Quelle erwähnt.

Weiterhin spricht für Philipps Argumentation, dass der Chorneubau bereits vor den Silberfunden am Schneeberg 1470 und damit vor Zwickaus wirtschaftlicher Blütezeit durchgeführt wurde. Spektakuläre Silberfunde am nahe gelegenen Schneeberg führten in den Jahren nach $1470 \mathrm{zu}$ einem bis dahin nicht gekannten Reichtum in Zwickau. ${ }^{33}$ Die vermutete Finanzierung des Chorneubaus durch die Zwickauer, präziser vermutlich durch den Rat und vor allem die ,fabrica ecclesiae der Marienkirche, könnte demzufolge gezielt übernommen worden sein und nicht, weil diese ohnehin über hohe Einnahmen verfügten.

Hohe Einnahmen durch die Silberfunde ermöglichten es 1479, einen wertvollen Altar des Nürnberger Künstlers Michael Wolgemut anzuschaffen und als neuen Hochaltar im nunmehr fertiggestellten Chorraum aufzustellen; diese Kosten in Höhe von 1400 Gulden wurden von der fabrica ecclesiae bestritten. ${ }^{34}$ Auf

31 Peter Schumann, Annalen der Stadt Zwickau (Ratsschulbibliothek Zwickau, Ms. 550); ähnlich auch ERnst Fabian, Die Oswald Lasanschen Annalen der Stadt Schwanfeld oder Zwickau von 1231-1534, in: Mitteilungen des Altertumsvereins für Zwickau und Umgegend 10 (1910), S. 1-68, hier S. 22.

32 Kirsten, Dom St. Marien (wie Anm. 2), S. 8; Traugott Wilhelm Hildebrand, Die Hauptkirche St. Mariä zu Zwickau, Zwickau o. J. [etwa 1841], S. 33. Die Inschrift lautet: Anno Domini MCCCCLIII. Diebus mensis Julii fundamentum bujus chori inchoatum fuit.

33 Zur Bedeutung des Bergbaus im Westerzgebirge vgl. Oswald Hoppe, Der Silberbergbau zu Schneeberg bis zum Jahre 1500, Diss. Freiberg 1908; Karl Hahn, Die ältesten Schneeberger Zehntrechnungen, in: NASG 53 (1932), S. 35-50; Adolf Laube, Studien über den erzgebirgischen Silberbergbau von 1470 bis 1546 (Forschungen zur mittelalterlichen Geschichte 22), Berlin 21976; Uwe Schirmer, Die Finanzen der Kurfürsten und Herzöge von Sachsen zwischen 1485 und 1547, in: Uwe John/Josef Mazerath (Hg.), Landesgeschichte als Herausforderung und Programm. Karlheinz Blaschke zum 70. Geburtstag, Stuttgart 1997, S. 259-284; Ders., Kursächsische Staatsfinanzen (14561656). Strukturen - Verfassung - Funktionseliten (Quellen und Forschungen zur sächsischen Geschichte 28), Stuttgart 2006.

34 StA Zwickau, III Z ${ }^{4 k}$, Nr. 70, Rechnungen Marienkirche, Bd. 1: 1441-1489, Nr. 2 (1476-1483), fol. 21v: XIIIJ C gulden meister Michel von Nürnbergk vor die taffel zcu machen mit sampt dem fürlon. Vgl. dazu auch Gerhard BeTz, Der Nürnberger Maler Michael Wolgemut (1434-1519) und seine Werkstatt. Ein Beitrag zur Geschichte der spätgotischen Malerei in Franken, Diss. phil. masch. Freiburg im Breisgau 1955, S. 204219; Der Zwickauer Wolgemut-Altar. Beiträge zu Geschichte, Ikonographie, Autorschaft und Restaurierung (Arbeitshefte des Landesamtes für Denkmalpflege Sachsen 11), hrsg. vom Landesamt für Denkmalpflege Sachsen, Dresden 2008. 
der Rückseite war eine Tafel angebracht, die das Kaufdatum des Altars, den Preis und die an dem Kauf Beteiligten nannte. ${ }^{35}$ Neben dem Zwickauer Hauptmann Martin Römer als Vermittler traten der Zwickauer Rat und Michael Wolgemut in Erscheinung.

Allerdings stellte Arnd Reitemeier in seiner Untersuchung über die spätmittelalterliche fabrica ecclesiae fest, dass die Patronatsherren zwar verpflichtet waren, für den Bau und die Unterhaltung der Kirche Sorge zu tragen, falls die Mittel der Kirchenfabrik nicht ausreichten, dass dies aber in den seltensten Fällen auch geschah. ${ }^{36}$ Insofern lassen sich die Überlegungen der Zwickauer in der Mitte des 15. Jahrhunderts zwar erahnen, zweifelsfrei belegen lassen sich ihre Bemühungen zum Kauf des Patronatsrechtes aber erst seit Ende des 15. Jahrhunderts. Bedenkenswert sind in diesem Kontext die Überlegungen von Michael Kirsten, der den Beginn des Chorumbaus auf ein gewachsenes Selbstbewusstsein der Stadt Zwickau in der Mitte des 15. Jahrhunderts zurückführt, das unter anderem aus dem Erwerb der hohen und niederen Gerichtsbarkeit 1444 resultierte. ${ }^{37}$

Der Beginn des Neubaus des Langhauses 1506 und damit die enge zeitliche Abfolge zwischen dem Kauf des Nominationsrechtes 1504 und dem Baubeginn legt einen Zusammenhang nahe. Die Grundsteinlegung wurde 1506 feierlich vollzogen, wie die Kirchenrechnungen jenes Jahres berichten. So wurden der Weihbischof eingeladen, drei Mahle im Wert von 4 Gulden ausgerichtet und insgesamt 4 Gulden und 18 Groschen an den Klerus, die Bediensteten der Marienkirche und die anwesenden Maurer und Steinmetzen gezahlt. ${ }^{38}$ Ähnlich wie 1453 wurde auch 1506 eine Gedenktafel gestiftet, auf der neben den Bürgermeistern Michael Rang und Urban Thiemer die beiden Kirchenmeister Hans Jacoff (Jacob) und Cuntz Knobloch genannt wurden. ${ }^{39}$

35 Kirsten, Dom St. Marien (wie Anm. 2), S. 24; Emil Herzog, Chronik der Kreisstadt Zwickau, Erster Teil, Zwickau 1839 (Reprint Stuttgart 1999), S. 96.

36 Arnd Reitemeier, Pfarrkirchen in der Stadt des späten Mittelalters. Politik, Wirtschaft und Verwaltung (Vierteljahrschrift für Sozial- und Wirtschaftsgeschichte, Beihefte 177), Stuttgart 2005, S. 474.

37 Michael Kirsten, Der Wolgemut-Altar in der Stadtkirche St. Marien zu Zwickau, in: Der Zwickauer Wolgemut-Altar (wie Anm. 34), S. 14-24, hier S. 15.

38 StA Zwickau, III Z ${ }^{4 k}$, Nr. 70, Rechnungen Marienkirche, Bd. 4: 1504-1508, Nr. 2 (1506-1507), fol. 72v; größere Mahlzeiten und Freigetränke für die beteiligten Handwerker gehörten traditionell zu einer Grundsteinlegung dazu, vgl. FrIEDRICH Weber, „... als sie den ersten stein gelegt“. Baubräuche im frühneuzeitlichen Siegen, in: Siegerland. Blätter des Siegerländer Heimat- und Geschichtsvereins 76 (1999), Heft 1, S. 2940, hier S. 29 f.

39 Hildebrand, St. Mariä (wie Anm. 32), S. 35: Im iare christi MCCCCCVI sontags exaudi ist gelegt der erste gruntstein, als dise kirch aufs new angefangen. Dy czeit die erbaren Michael Rang und Urban Thimer burgermeister, Hans Jacof und Cuntz Knobloch alterleut gewest. Es war in anderen Städten durchaus üblich, dass auch das geistliche Personal und doch zumindest der Pfarrer auf dem Grundstein verzeichnet wurden, wie zwei Grundsteinplatten aus der Leipziger Nikolaikirche von 1513 belegen, vgl. ENNO BüNZ, Zwei Grundsteinplatten aus der Nikolaikirche, in: Volker Rodekamp (Hg.), Leipzig original. Stadtgeschichte vom Mittelalter bis zur Völkerschlacht, Katalog zur 
Das großangelegte Bauvorhaben des Zwickauer Rates traf in der Gemeinde auf Zustimmung, was sich anschaulich an der Steigerung des eingelegten ,Tafelgeldes am Kirchweihtag der Marienkirche demonstrieren lässt. Während des Gottesdienstes schritt üblicherweise der Küster durch die Reihen der Gottesdienstbesucher und sammelte Spenden mithilfe einer Opfertafel ein. ${ }^{40}$ Im Allgemeinen war der Tag der Weihe der Pfarrkirche einer der Tage, an dem die Zahl der Kirchbesucher am größten und mithin auch das Spendenaufkommen am höchsten war. Die Vermutung liegt nahe, dass sich gerade an einem solchen Tag, der unmittelbar mit der Kirche als Bauwerk in Verbindung steht, die Identifikation der Pfarrgemeinde mit dieser besonders prominent äußert. Dies bestätigt sich bei einer Betrachtung der Kirchenrechnungen der Marienkirche aus den Jahren 1502 bis 1514 (Abb. 1): Direkt nach 1506 stieg das Spendenaufkommen am Kirchweihtag stark an, sank bis 1514 jedoch auf den Stand vor den Bauarbeiten ab.

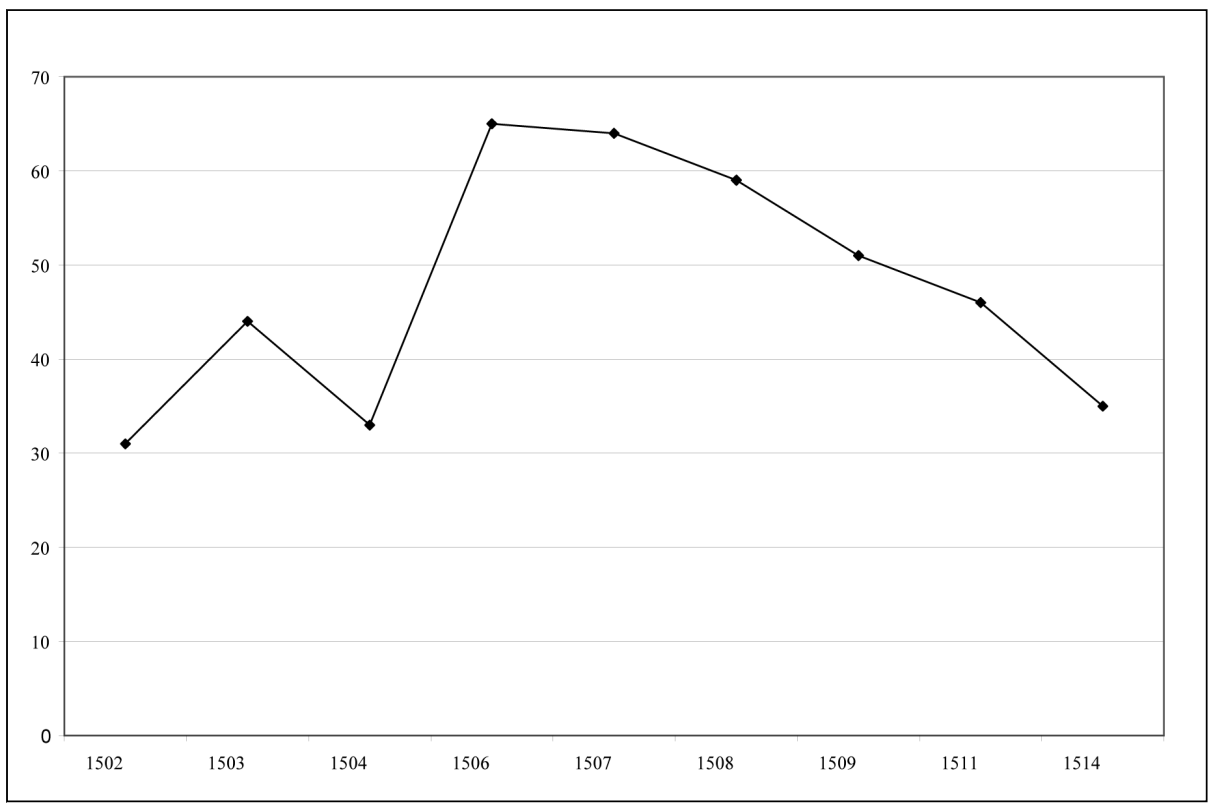

Abb. 1: Tafelgeld am Kirchweibtag (in Groschen).

Der oben dargestellten These von der ,Signalfunktion' des Neubaus des Langhauses nach 1506 stehen jedoch die ebenfalls bereits geschilderten Vorbereitungen, Gespräche und die Einholung von Gutachten von überregional bekannten Bau-

Dauerausstellung des Stadtgeschichtlichen Museums im Alten Rathaus, Teil I, Leipzig 2006, S. $67 \mathrm{f}$.

40 Heinrich Dormeier, Kirchenjahr, Heiligenverehrung und große Politik im Almosengefällbuch der Nürnberger Lorenzpfarrei, in: Mitteilungen des Vereins für Geschichte der Stadt Nürnberg 84 (1997) S. 1-60, hier S. 4. 
meistern bereits im letzten Viertel des 15. Jahrhunderts entgegen. Der Erfolg der Zwickauer Anstrengungen war nämlich bis kurz vor dem Erwerb des Nominationsrechtes keineswegs sicher, da das Kloster Eisenberg vermutlich durch eine wirtschaftliche Notlage zum Verkauf gezwungen wurde. ${ }^{41}$ Noch 1504 schlugen Verhandlungen zwischen den Zwickauern und dem Kloster Eisenberg in Zeitz fehl, weil sich die beiden Parteien nicht über die Verteilung der Kosten für die Einholung der entsprechenden Privilegien an der Kurie und beim Landesherrn einigen konnten. ${ }^{42}$ Als die Verhandlungen in Zwickau fortgesetzt wurden, stimmte der Zwickauer Rat der Kostenübernahme nur unter der Bedingung zu, dass sich die Eisenberger Nonnen tatsächlich an den Kaufvertrag halten würden. ${ }^{43}$ Auch legt die Einbettung der Langhauserweiterung in die vorhergehenden Baumaßnahmen am Turm und dem Chor und insbesondere die Fertigstellung der Bauten an Sakristei und Sängerempore 1507 nahe, ${ }^{44}$ dass der Beginn der Bauarbeiten am Langhaus durch technische und architektonische Gegebenheiten festgelegt wurde.

Eine ,Symbolwirkung' des Bauvorhabens ist folglich vorsichtig zu beurteilen, da die Planung dieses Neubaus aufgrund langfristiger Überlegungen geschah, was jedoch dennoch zu Schurrs Argumentation passt, der den Prozess der Übernahme des Patronats als in vielen Fällen langfristiges Ringen zweier Kräfte sieht, währenddessen „Stück für Stück in schmalen Tranchen“ die kirchlichen Rechte erstritten wurden. ${ }^{45}$

\section{Die Vorbildwirkung Nürnbergs und der erzgebirgischen Bergstädte}

Die jahrzehntelange Baugeschichte der Zwickauer Marienkirche wurde in der Geschichtswissenschaft recht unterschiedlich bewertet. Kurt Gerstenberg beispielsweise beurteilte 1913 in seinem gleichermaßen bekannten wie umstrittenen Werk „Die deutsche Sondergotik“ die häufigen Änderungen des Bauplanes der Marienkirche durchaus positiv: „Die Baugeschichte der Zwickauer Marienkirche liefert den Beweis dafür, wie das wachsende Raumgefühl ständig die alte Schale sprengt.“ 46 Gerstenberg zufolge habe das „Raumgefühl ein so mächtiges Schwellen des Raumes verlangt“, dass daraus die erwähnten Modifikationen des Bauvorhabens resultierten. Insbesondere der weit auseinandergezogene Triumphbogen der Marienkirche lasse die „raumweitende Kraft der Sondergotik“ erkennen. ${ }^{47}$

41 Vgl. dazu Armin Basedow, Die letzten Jahrzehnte des Nonnenklosters zu Eisenberg, in: Sachsen-Altenburgischer vaterländischer Geschichts- und Hauskalender 94 (1927), S. 104-106.

42 StA Zwickau, A*A II 18, Nr. 38 (2).

43 Ebd.

44 KüHN, Baugeschichte (wie Anm. 5), S. 9.

45 Schurr, Architektur (wie Anm. 23), S. 264.

46 Kurt Gerstenberg, Deutsche Sondergotik. Eine Untersuchung über das Wesen der deutschen Baukunst im späten Mittelalter, Darmstadt 21969, S. 169.

47 Ebd. 
Demgegenüber schätzte Werner Müller 1997 das Phänomen weit negativer ein, da seiner Meinung nach besonders die langwierigen Diskussionen des Zwickauer Rates vor dem Bau des Daches den Eindruck einer „Versammlung von Leuten [hinterließen], die, in ihrem künstlerischen Urteil ebenso inkompetent wie unsicher, vor allem darauf bedacht waren, einen möglichst modernen, aber an anderer Stelle bereits anerkannten Entwurf zu wählen. “48

Tatsächlich stellt sich die Frage nach der Beeinflussung der Zwickauer Ratsherren durch den Kirchenbau in anderen Städten. Bezogen auf den von Martin Warnke in die Diskussion eingebrachten Begriff des „künstlerischen Anspruchsniveaus“, worunter er den „Umfang baulicher oder künstlerischer Leistungen“ verstand, „der es in einer geschichtlichen Epoche Individuen oder Gruppen ermöglicht, ihre soziale Stellung und Funktion sichtbar zu bestimmen oder zu erfahren", ${ }^{49}$ kommen für die verschiedenen Bauphasen unterschiedliche Bezugspunkte in Frage. Warnke zufolge steht ein Kirchengebäude mit anderen Bauten, die sowohl innerhalb als auch außerhalb der Stadt liegen konnten, in Konkurrenz, was wiederum das Anstellen von Rangvergleichen zwischen Städten und Institutionen fördert. Wendet man seine Thesen auf Zwickau an, dann erscheint die Reichsstadt Nürnberg Mitte des 15. Jahrhunderts als entsprechendes ranghöheres Vorbild für den Kirchenbau. Nürnberg stand traditionell in einem engen Verhältnis zur ehemaligen Reichsstadt Zwickau, die bis 1307 neben Altenburg und Chemnitz eines der Zentren des Reichsterritoriums Pleißenland bildete. Dass der reichsstädtische Anspruch der Zwickauer auch Jahrzehnte nach dem Übergang Zwickaus an die Wettiner nach 1307 noch lebendig war, zeigt beispielsweise die Teilnahme des Zwickauer Bürgermeisters am Städtetag in Nürnberg im Jahr 1399.50 Die geistige Nähe zu Nürnberg wird unter anderem daran sichtbar, dass einige Nürnberger Künstler im Auftrag der Zwickauer tätig waren. Beispielsweise wurde der heute noch erhaltene wertvolle Hauptaltar der Marienkirche von Michael Wolgemut aus Nürnberg erstellt. Auch der Schöpfer des Heiligen Grabes in der Marienkirche,

48 Werner Müller/Norbert Quien, Von deutscher Sondergotik. Architekturphotographie, Computertomographik, Deutung (Saecula Spiritualia 33), Baden-Baden 1997, S. 74 .

49 Martin Warnke, Bau und Überbau. Soziologie der mittelalterlichen Architektur nach den Schriftquellen, Frankfurt a. M. 1976, S. 13; zur Kritik an Warnkes Modell vgl. Martial Staub, Stifter als „Unternehmer“. Frömmigkeit und Innovation im späteren Mittelalter am Beispiel Nürnbergs, in: Klaus Schreiner/Marc Müntz (Hg.), Frömmigkeit im Mittelalter. Politisch-soziale Kontexte, visuelle Praxis, körperliche Ausdrucksformen, München 2002, S. 155-178, hier S. 160.

50 Julius Weizsäcker (Hg.), Deutsche Reichstagsakten unter König Wenzel, 3. Abteilung: 1397-1400 (Deutsche Reichstagsakten 3), Göttingen 21956, S. 145, Nr. 102. Auch die Korrespondenz zwischen Kurfürst Friedrich dem Weisen und der Reichsstadt Nürnberg illustriert beispielhaft deren engen wirtschaftliche, politische und geistige Verbindungen, vgl. Sina Westphal, Die Korrespondenz zwischen Kurfürst Friedrich dem Weisen von Sachsen und der Reichsstadt Nürnberg. Analyse und Edition (Kieler Werkstücke E/10), Frankfurt a. M. 2011. 
Michael Heuffner, stammte aus Eger und gehörte der Nürnberger Schule an. ${ }^{51}$ Insbesondere der Bau des Chores der Marienkirche könnte vom Vorbild Nürnbergs beeinflusst worden sein. Die dortige Lorenzkirche erhielt zwischen 1439 und 1454 unter der Leitung des aus Rothenburg berufenen Konrad Heinzelmann einen neuen spätgotischen Hallenchor mit einem Drei-Achtel-Schluss, der mit einiger Wahrscheinlichkeit zumindest einen Einfluss auf den Bau der Marienkirche hatte. ${ }^{52}$ Einschränkend muss jedoch bemerkt werden, dass sich auch einige Gemeinsamkeiten der Marienkirche mit den Chören von St. Martin in Amberg und St. Michael in Schwäbisch-Hall feststellen lassen. ${ }^{53}$

Bereits einige Jahrzehnte nach dem Bau des Chores, als der Neubau des Langhauses anstand, war hingegen die Konkurrenz zu anderen erzgebirgischen Städten ausschlaggebend für viele Entscheidungen. Zwickau befand sich zu Beginn des 16. Jahrhunderts zwar in einer sehr guten wirtschaftlichen Ausgangslage, jedoch zeichneten sich bereits kommende Probleme ab, die aus dem Verlust des Einflusses auf die Bergstädte, vor allem auf Schneeberg, resultierten. ${ }^{54}$ Um 1530 geriet die Stadt in eine schwere wirtschaftliche Krise, unter anderem da sich die größere Unabhängigkeit Schneebergs von Zwickauer Produkten und eine sich verstärkende Inflation negativ auswirkten. ${ }^{55}$ Die bereits beschriebene Reise des Zwickauer Baumeisters Caspar Teicher nach Schneeberg, um das neu errichtete Dach der Wolfgangskirche zu inspizieren, demonstriert, wie sich die Parameter in Zwickau verschoben hatten. Im Allgemeinen lässt sich der Ausbau der Marienkirche in den Komplex der unterschiedlichen Ausprägungen der obersächsischen Spätgotik einreihen, deren jeweilige wechselseitige Einflüsse sich im Einzelnen nur schwer belegen lassen.56 Karl Weißbach unternahm in seiner Untersuchung der Baugeschichte der Marienkirche dennoch den Versuch, dem Einfluss anderer Bauvorhaben auf die Marienkirche nachzugehen. ${ }^{57}$ Beim Bau des Langhauses erhielten die durch das Einziehen der Strebepfeiler entstehenden umlaufenden Emporen Kanzelausbauten um die oberen Teile der Strebepfeiler, die ähnlich auch im Frei-

51 Karant-Nunn, Zwickau in Transition (wie Anm. 8), S. 200.

52 Art. Nürnberg. St. Lorenz, in: Georg Dehio, Handbuch der deutschen Kunstdenkmäler, Bayern I: Franken, bearb. von Tilman Breuer u. a., München/Berlin 21999, S. 698-716; Baier, Dom St. Marien (wie Anm. 8), S. 5; Brugmann, Zwickau (wie Anm. 8), S. 1082; Norbert Nussbaum, Deutsche Kirchenbaukunst der Gotik, Darmstadt 21994, S. 245.

53 Weissbach, Marienkirche (wie Anm. 7), S. 44.

54 Helmut BräUer, Wider den Rat. Der Zwickauer Konflikt 1516/17 (Zwickauer Arbeits- und Forschungsberichte. Kulturgeschichtliche Beiträge, Beiheft 1), Leipzig 1999, S. 37-40; Karant-Nunn, Zwickau in Transition (wie Anm. 8), S. 66-90.

55 Vgl. Karant-Nunn, Zwickau in Transition (wie Anm. 8), S. 66-90.

56 Vgl. Weissbach, Marienkirche (wie Anm. 7); Magirius, Schneeberg (wie Anm. 20); Hermann Meuche, Anmerkungen zur Gestalt der sächsischen Hallenkirchen um 1500, in: Aspekte zur Kunstgeschichte von Mittelalter und Neuzeit. Karl Heinz Clasen zum 75. Geburtstag, Weimar 1971, S. 167-190; Nussbaum, Deutsche Kirchenbaukunst (wie Anm. 52); Matthias Barth, Baukunst des Mittelalters in Sachsen, Rostock 1998. 
Julia Kahleyß

berger Dom vorkommen. ${ }^{58}$ Weißbach nahm an, dass die gesamte Emporenanlage des Domes von den Zwickauern kopiert wurde, da im Jahr 1497 der Parlierer von Freiberg nach Zwickau bestellt wurde, um das Langhaus zu begutachten. ${ }^{59}$ Große Ähnlichkeiten stellte Weißbach auch zwischen dem Langhaus der Marienkirche und der Stadtkirche von Jena fest, was sich wohl aus der Herkunft des 1507 verstorbenen Baumeisters Peter Heierliß erklären lässt.60 Die Baumeister der Annenkirche und der Stadtkirche in Brüx befassten sich mit dem Gewölbe der Marienkirche, wobei der Plan für das Gewölbe wahrscheinlich von Caspar Teicher stammt. Wessen Ideen er später aufgriff und für den Bau der Marienkirche abwandelte, lässt sich nicht mehr nachvollziehen. ${ }^{61}$

\section{Die wirtschaftlichen Voraussetzungen für die Gestaltung des sakralen Raumes: die Silberfunde nach 1470 und ibre Wirkung auf die Kirchenfabrik}

Ein weiterer wichtiger Einflussfaktor auf die Gestaltung des Kirchenraumes der Marienkirche war die wirtschaftliche Situation der Kirchenfabrik. Nach 1470 brachten die Silberfunde am nahe Zwickau gelegenen Schneeberg einen starken wirtschaftlichen Aufschwung, der nicht nur einzelnen ,Fundgrübnern', sondern auch der Kirchenfabrik der Marienkirche steigende Einnahmen bescherte. Die Ursprünge der fabrica ecclesiae lagen in der treuhänderischen Verwaltung von Schenkungen, die allein für das Kirchengebäude bestimmt waren, durch Laien aus der Pfarrgemeinde.62 Die in der laikalen Verwaltung entstehenden Kirchenrechnungen bilden eine wichtige Quellengattung, die vielfältige Einblicke in das kirchliche Alltagsleben bietet. ${ }^{63}$ Auch auf die Frage, welchen Einfluss die wirtschaftlichen Bedingungen auf den Bau einer Kirche hatten, lassen sich mithilfe dieser seriellen Quellen Antworten finden. Die Kirchenrechnungen der Marienkirche liegen ab 1441 vor, wobei eine große Überlieferungslücke zwischen 1448 und 1476

58 Ebd., S. 45.

59 Ebd.

60 Ebd., S. 46.

61 Ebd., S. 47; Kirsten, Dom St. Marien (wie Anm. 2), S. 12.

62 Zur Entwicklung der fabrica ecclesiae vgl. Sebastian Schröcker, Die Kirchenpflegschaft. Die Verwaltung des Niederkirchenvermögens durch Laien seit dem ausgehenden Mittelalter (Görres-Gesellschaft. Veröffentlichung der Sektion für Rechts- und Staatswissenschaften 67), Paderborn 1934; Wolfgang Schöller, Die rechtliche Organisation des Kirchenbaues im Mittelalter vornehmlich des Kathedralbaues. Baulast Bauherrenschaft - Baufinanzierung, Diss. phil. Marburg a. d. Lahn, Köln/Wien 1989, S. 345-359; Reitemeier, Pfarrkirchen (wie Anm. 36); Uwe Schirmer, Unerschlossene Quellen zur Reformationsgeschichte: Kirchenrechnungen aus dem ernestinischen Kursachsen (1514-1547), in: Winfried Müller (Hg.), Perspektiven der Reformationsforschung in Sachsen. Ehrenkolloquium zum 80. Geburtstag von Karlheinz Blaschke (Bausteine aus dem Institut für Sächsische Geschichte und Volkskunde 12), Dresden 2008, S. 107-124.

63 Vgl. den Forschungsüberblick zur fabrica ecclesiae bei Reitemeier, Pfarrkirchen (wie Anm. 36), S. 22 f. 
zu konstatieren ist. ${ }^{64}$ Durch die Silberfunde am Schneeberg konnte die Kirchenfabrik im späten 15. Jahrhundert große Einnahmen verbuchen, wie die Auswertung des Rechnungszeitraumes 1476 bis 1483 (Abb. 2) zeigt.

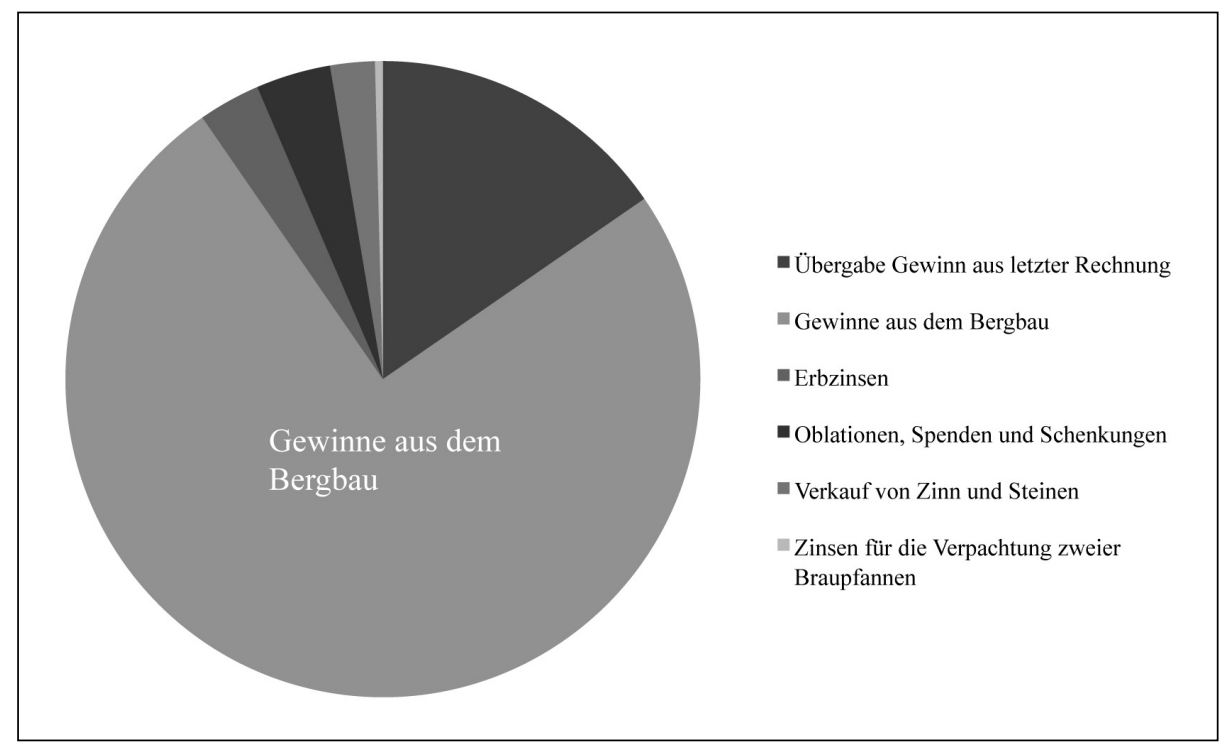

Abb. 2: Einnabmen der Kirchenfabrik im Zeitraum 1476-1483.

Nach Abzug der allgemeinen Betriebsausgaben blieb genügend Geld übrig, um die Ausstattung der Kirche zu erneuern. Dies beinhaltete nicht nur den Kauf von liturgischen Geräten, Gewändern etc., sondern auch von wertvollen Kunstgegenständen, die bis heute eine der Attraktionen der Marienkirche bilden. Neben dem oben beschriebenen Hochaltar wurden fünf Bilder, ein Kruzifix, ein kristallenes Kreuzreliquiar, ein silbernes Kreuz und weiteres goldenes und silbernes liturgisches Gerät gekauft. ${ }^{65}$ Außerdem wurde eine von Nikolaus von Merseburg hergestellte neue Orgel angeschafft. ${ }^{66}$

Die Gewinne aus dem Bergbau brachen nach einigen Jahren ein. Da bis 1506 trotz sinkender Einkünfte jedoch immer noch die Einnahmen die Ausgaben übertrafen und die Zwickauer bereits Ende des 15. Jahrhunderts Überlegungen zur Gestaltung des neuen Langhauses angestellt hatten, orientierte sich die Ausführung des Neubaus wohl an den hohen Einkünften der vorhergehenden Jahrzehnte.

64 StA Zwickau, III Z ${ }^{4 k}$, Nr. 70, Rechnungen Marienkirche, Bd. 1: 1441-1489; Bd. 2: 1490-1500; Bd. 3: 1500-1504; Bd. 4: 1504-1508; Bd. 5: 1508-1510; Bd. 6: 1510-1520; Bd. 7: 1521-1534. Es sei an dieser Stelle auf eine von der Verfasserin erstellte Edition ausgewählter Zwickauer Kirchenrechnungen hingewiesen (im Druck).

65 Kirsten, Dom St. Marien (wie Anm. 2), S. 36.

66 StA Zwickau, III Z4k, Nr. 70, Rechnungen Marienkirche, Bd. 1: 1441-1489, Nr. 2 (1476-1483), fol. 21r. 


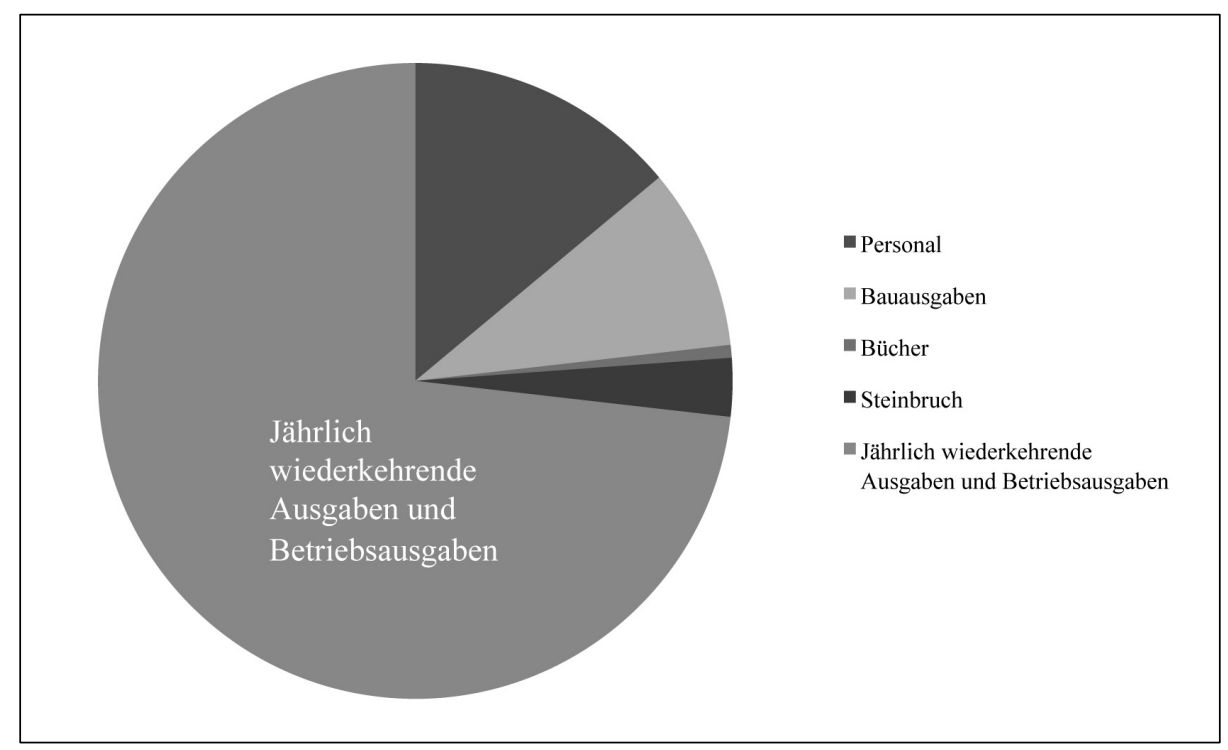

Abb. 3: Ausgaben der Kirchenfabrik im Zeitraum 1503/04.

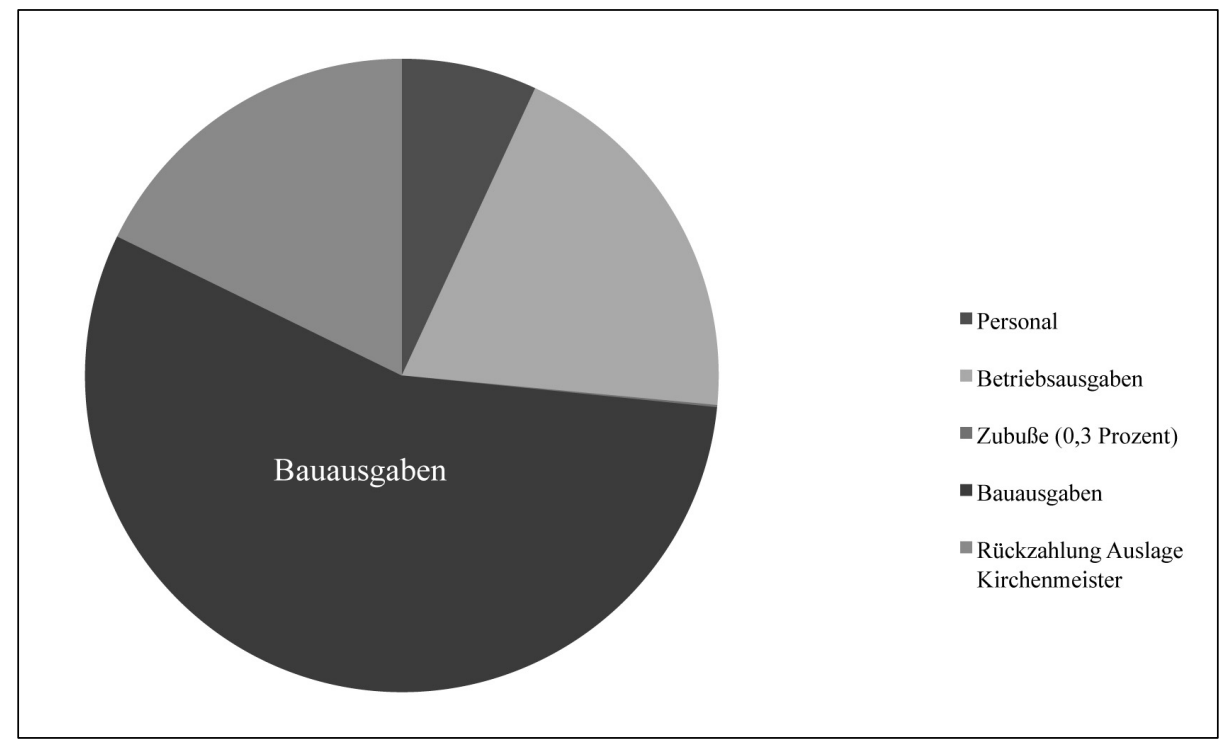

Abb. 4: Ausgaben der Kirchenfabrik im Zeitraum 1512/13.

Tatsächlich konnte die Kirchenfabrik steigende Einnahmen verzeichnen, gleichzeitig gaben die Kirchenmeister jedoch dauerhaft mehr für den Bau aus, als sie einnahmen.

Wie hoch die finanzielle Belastung durch den Neubau des Langhauses für die Kirchenfabrik tatsächlich war, zeigen die Ausgaben der Kirchenfabrik im Rech- 
nungsjahr 1512/13 (Abb. 4). 55 \% der Ausgaben wurden nur für den Neubau des Kirchenschiffs aufgewendet. Es handelte sich um Zahlungen an Steinmetze, Zimmerleute, sonstige Handwerker und Tagelöhner sowie für Baumaterial. Sonstige Reparaturen des Kirchengebäudes wurden unter Betriebsausgaben subsumiert. 18 \% der Ausgaben wurden für die Rückzahlung der Auslage des Kirchenmeisters im vorhergehenden Rechnungszeitraum verwendet. Sie waren wahrscheinlich auch dem Kirchenbau geschuldet, sodass nach Addition dieser Rückzahlung 73 \% der Ausgaben für den Neubau geleistet wurden. Der Vergleich fällt umso eindrucksvoller aus, wenn daneben die Ausgabenverteilung im Rechnungsjahr 1503/04 (Abb. 3) betrachtet wird. In diesem Jahr nahmen die jährlich wiederkehrenden Zahlungsverpflichtungen und Betriebsausgaben den weitaus größten Raum ein.

\section{Die Rolle des Stadtrates als Bauberr und des Baumeisters}

Die Schwierigkeiten bei der Finanzierung des Neubaus der Marienkirche werfen die Frage nach der Verantwortung des Stadtrates als (Mit-)Bauherr für den Kirchenbau auf. Generell muss die Rolle eines Bauherrn bei der Planung und Ausführung eines Bauvorhabens im Mittelalter differenziert beurteilt werden. Laut Warnke nahm dieser seit dem 11. Jahrhundert zwar eine wichtige Position ein, die praktische Ausführung lag aber in der Verantwortung der Bauverwalter. ${ }^{67}$ In ähnlicher Weise stellte Bandmann fest, dass sich zwischen dem 13. und dem 15. Jahrhundert die Bedeutung des Bauherrn für das Bauwerk verminderte, da ein eigener Architektenstand und ein selbstständiges Hüttenwesen entstanden seien. ${ }^{68}$ Andererseits vertrat Philipp die Ansicht, dass die „Stellung des Baumeisters der Spätgotik zwar relativ autonom" gewesen sei, aber er sich trotzdem nach den Vorstellungen seiner Auftraggeber habe richten müssen. Deshalb sieht Philipp jedes Kirchengebäude als „Kompromiss“ zwischen dem Baumeister und dem Auftraggeber. ${ }^{69}$ Ausgehend von diesen Vorgaben wird im Folgenden die direkte Einflussnahme des Zwickauer Rates, die nicht mittelbar durch die dem Rat angehörenden Kirchenmeister vorgenommen wurde, auf die Gestaltung des Kirchenbaus der Marienkirche betrachtet werden. Der Zwickauer Rat beschäftigte sich nicht nur mit Grundsatzentscheidungen, zum Beispiel über den Grundriss des Gebäudes

67 Vgl. Warnke, Bau und Überbau (wie Anm. 49), S. 102 f., vgl. zu dieser Problematik auch die Beiträge in Bruno Klein/Katja Schröck/Stefan Bürger (Hg.), Kirche als Baustelle. Große Sakralbauten des Mittelalters, Köln/Weimar/Wien 2013.

68 Günter Bandmann, Mittelalterliche Architektur als Bedeutungsträger, Berlin 101994, S. 47.

69 Philipp, Pfarrkirchen (wie Anm. 22), S. 12. Ähnlich sieht dies Corine Schleif in Bezug auf die Objektstiftungen in der Nürnberger Lorenzkirche, vgl. Corine SchleIf, Donatio et Memoria. Stifter, Stiftungen und Motivationen an Beispielen aus der Lorenzkirche in Nürnberg, Diss. phil. München 1990, S. 61 f. 
oder die Beauftragung geeigneter Baumeister, sondern die Ratsherren trafen häufiger auch ganz konkrete Entscheidungen, die die Ausführung des Bauvorhabens beeinflussten und in den Ratsprotokollen festgehalten wurden. Beispielsweise wurde über die Frage der Reparatur oder der völligen Abtragung des Gewölbes entschieden, ${ }^{70}$ es wurde der Beschluss gefasst, die alte Empore eine Zeit lang unter der neuen Empore stehen zu lassen ${ }^{71}$ oder dem Baumeister Caspar Teicher nach Abschluss der Arbeiten ein Holzgestell zu überlassen. ${ }^{72}$ Im Jahr 1529 beschloss der Rat sogar, dass der Kirchenmeister der Marienkirche ein Seil aus dem Steinbruch erhalten sollte, weil das der Marienkirche beim Bau des Gewandhauses zerrissen sei. ${ }^{73}$ Der Rat traf auch Vorsorge für die Finanzierung des Kirchenbaus, ${ }^{74}$ was 1523 zur Einstellung der Baumaßnahmen führte, da der Rat durch viele neue, kostspielige Bauten in Zwickau in Geldschwierigkeiten geraten war. ${ }^{75}$ 1528/29 konnte die Kirchenfabrik von einer großzügigen Anleihe des Rates über 400 Gulden profitieren. Dieses Geld hatte der Rat in Altenburg angelegt, aber angesichts der Ausgaben für das neue Kirchendach ausgelöst und der Kirchenfabrik zukommen lassen. ${ }^{76}$

Bei der Beurteilung der Frage, wie sich die Verantwortungsteilung zwischen Baumeister und Bauherr gestaltete, bezeugen die vorliegenden Quellen die Vorrangstellung des Rates. Bei vielen in den Ratsprotokollen dokumentierten Entscheidungen wurden die Baumeister nicht erwähnt. Dennoch müssen sie aber doch über genügend Entscheidungsspielraum verfügt haben, denn die Ratsherren waren in der Mehrzahl keine Fachleute. Im Einzelfall war die Entscheidungsfindung wohl ähnlich, wie 1523 in den Protokollen festgehalten wurde: Als darüber entschieden werden musste, ob die vorderen Pfeiler abgetragen und neu errichtet werden sollten, unterbreiteten Meister Caspar (Teicher) und Meister Friedrich dem Rat Lösungsvorschläge, woraufhin dieser sich für eine Variante entschied.77 Auf diese Weise wurde der Rat jedoch nicht zu einem Instrument seines Baumeisters, denn es wurden immer wieder andere Baumeister zur Klärung spezieller Fragen nach Zwickau gebeten, beispielsweise der Baumeister aus Annaberg78 oder der Turmsteiger aus Altenburg. ${ }^{79}$ 1511, als sich im alten Gewölbe des Langhauses einige Risse zeigten, ließ der Rat gleich fünf fremde Werkmeister rufen. Caspar Teicher hatte die Risse zwar als ungefährlich eingeschätzt, dennoch bestand der

StA Zwickau, IIIx, 59b/26, $13 \mathrm{~b}$.

StA Zwickau, IIIx, 59b/133, 29b.

2 StA Zwickau, IIIx, 64/159, 60b.

73 StA Zwickau, IIIx, 63/58, 83a.

74 StA Zwickau, III b1, 23b.

75 Hildebrand, St. Mariä (wie Anm. 32), S. 38; StA Zwickau, IIIx, 61/47, 34 b. 
Rat darauf, eldere und kunstfertige Baumeister kommen und den Bau begutachten zu lassen. ${ }^{80}$

\section{Der Einbau von Kirchengestübl im Langhaus}

Ein deutliches Zeichen für den Einfluss des Rates in der städtischen Pfarrkirche war die Anbringung eines Ratsgestühles. ${ }^{81}$ Anders als in anderen Städten, beispielsweise Stralsund, ${ }^{82}$ hat sich aus dem Spätmittelalter kein Ratsgestühl in der Marienkirche erhalten. Das heute aufgestellte Ratsgestühl stammt aus dem 17. Jahrhundert. ${ }^{83}$ Dagegen datiert das repräsentative Chorgestühl, welches sich auch heute wieder im Chorumgang befindet, wahrscheinlich aus der Mitte des 15. Jahrhunderts. ${ }^{84}$

Kirchenstühle für Laien erscheinen in Zwickau erst Ende des 15. Jahrhunderts in den Kirchenrechnungen. ${ }^{85}$ Im Zuge der Baumaßnahmen des 15. und 16. Jahrhunderts wurden mehrere neue Stühle in der Kirche angebracht. ${ }^{86}$ Vor dem Einbau von Kirchenstühlen im Spätmittelalter gab es im Allgemeinen keine Sitzgele-

80 StA Zwickau, Ratsprotokolle I (1510/11), fol. 13r: Sonnabents fur Esto mibi 1511 das gewelb zu unsr lieben frawhen, dyweill sich das besorgsam und gebrechlich eraignt, ab man das abtragen wolde, zuvor an hanthwergr vnd gemeyn zu gelangen laßen; fol. 13v: Sonnabend nach Esto mibi [8. März 1513], Es ist auch durch bede rhete, hantberger vnd gemainden fur gut angesehn, das man uffs radts kostung uber dy bsichtigung Caspar Teichers, dy er am mangelhafftigem gewelb der pffarkirchen gthann, etzliche andre, eldere und kunstreicher baumaister ruffenn und dy selbn auch bsichtigen lasse, ap sich das gewelb uff lenger Zceitt leiden mochte, adder op man dasselbe abtragen lassen sollte.

81 Dietrich W. Poeck, Zahl, Tag und Stuhl. Zur Semiotik der Ratswahl, in: Frühmittelalterliche Studien 33 (1999), S. 396-427, hier S. 420-422; AnTJe Diener-Staeckling, Der Himmel über dem Rat. Zur Symbolik der Ratswahl in mitteldeutschen Städten (Studien zur Landesgeschichte 19), Halle a. d. Saale 2008, S. 225-227.

82 Sabine-Maria Weitzel, Die mittelalterliche Ausstattung von St. Nikolai in Stralsund. Überlegungen zur Funktion, Bedeutung und Nutzung einer hansestädtischen Pfarrkirche, in: Felix Biermann/Manfred Schneider/Thomas Terberger (Hg.), Pfarrkirchen in den Städten des Hanseraums (Archäologie und Geschichte im Ostseeraum 1), Rahden/ Westfalen 2006, S. 101-112, hier S. 105.

83 Kirsten, Dom St. Marien (wie Anm. 2), S. 46 f.

84 Die Existenz eines Chorgestühls weist in einer Pfarrkirche auf eine veränderte, nunmehr stiftsähnliche Liturgie hin, die den Klerus im gemeinsamen Hochamt in der Kirche versammelte, vgl. WeitzeL, Stralsund (wie Anm. 82), S. 107.

85 Ein vorreformatorisches Stuhlregister, wie es beispielsweise aus der Baseler Pfarrkirche St. Peter überliefert ist, hat sich in Zwickau nicht erhalten, vgl. dazu Gabriela Signori, Umstrittene Stühle. Spätmittelalterliches Kirchengestühl als soziales, politisches und religiöses Kommunikationsmedium, in: Zeitschrift für Historische Forschung 29 (2002), S. 189-213, hier S. 194 f. Die ältesten Stuhlregister der Zwickauer Kirchen St. Marien und St. Katharinen stammen aus den Jahren 1554 bis 1569, vgl. StA Zwickau, Kaland 2, Nr. 10.

86 StA Zwickau, III Z4k, Nr. 70, Rechnungen Marienkirche, Bd. 1: 1441-1489, Nr. 3 (1483-1486), fol. 36v: geben dem tischer von den frawen stulen zcu machen yn der kirchen. 
genheit für die Gemeinde. In vielen Kirchen wurden zunächst einzelne Plätze an Privatpersonen vermietet, die dann darauf einen Kirchenstuhl aufstellen durften, erst später wurden auch Kirchenstühle und Plätze auf den Bänken durch die Kirchenmeister verkauft. Damit erlangten die Käufer das dauerhafte Recht zur Nutzung ihres Kirchenstuhles, während die Kirchenmeister für die Pflege und Wartung des Gestühls verantwortlich blieben. ${ }^{87}$

Ähnlich war die Vergabe von Kirchenstühlen auch in Zwickau geregelt. Anfang des 16. Jahrhunderts lassen sich einige Belege für den Kauf eines Standes in der Marienkirche finden. ${ }^{88}$ Seit 1521 wurden vermehrt Plätze im Kirchengestühl verkauft: beispielsweise erwarben in dem halben Jahr zwischen Michaelis 1521 und Walpurgis 1522 Michel von Mila, die Hans Jacoffin und die Silberbrennerin einen Stand. ${ }^{99}$ Nach Einführung der Reformation setzte sich der Verkauf fort. So erwarben im Jahr 1528 der Organist, die Hans Appelin, die Herrewelin, ${ }^{90}$ Marcus Schusters Tochter und Caspar Jorgen einen Stuhl. ${ }^{91}$ Die Preise schwankten dabei zwischen fünf Groschen und einem Gulden. Die wenigen überlieferten Namen erlauben keine Aussage über eine anzunehmende Abbildung der sozialen Hierarchie Zwickaus in der Marienkirche mittels der verkauften Kirchenstühle. ${ }^{92}$ Dennoch lassen sich einige der erwähnten Käufer der Kirchenstühle der Zwickauer Oberschicht zuordnen. Die Hans Jacoffin war wahrscheinlich die Witwe von Hans Jacoff, eines 1506 von einem Bauern erschlagenen Ratsherrn und Kirchenmeisters der Marienkirche. ${ }^{93}$ Michel von Mila (Mylau) war zeitweise Ratsherr, Bürgermeister, Stadtvogt und einer der Vorsteher des Gemeinen Kastens. ${ }^{94}$ Caspar Jorgen war einer der Viermeister der Weber, der 1522 als verdächtiger Hundsgesser

87 Reitemeier, Pfarrkirchen (wie Anm. 36), S. 268, 465; Adolf Reinle, Die Ausstattung deutscher Kirchen im Mittelalter. Eine Einführung, Darmstadt 1988, S. 67 f.; PAUL Hinschius, System des katholischen Kirchenrechts mit besonderer Rücksicht auf Deutschland, Bd. 4, Berlin 1888 (Reprint Graz 1959), S. 341 f.; dagegen verweist Signori darauf, dass sich bereits ab dem 13. Jahrhundert die Hinweise auf Laienstühle in einzelnen Kirchen mehrten, vgl. SignORI, Umstrittene Stühle (wie Anm. 85), S. 190.

88 StA Zwickau, III Z ${ }^{4 k}$, Nr. 70, Rechnungen Marienkirche, Bd. 5: 1508-1510, Nr. 5 (1510), fol. 14r: X g VI d von Symon von Milaw entpfangen fur swertfegers standt in der kirchen. [...] II fl III g entpfangen von den newen gesatzten stulen bey dem altar sancti Martini $[\ldots]$.

89 StA Zwickau, III Z ${ }^{4 k}$, Nr. 70, Rechnungen Marienkirche, Bd. 7: 1521-1534, Nr. 1 (1521-1522), fol. 1v; Nr. 3 (1522-1523), fol. 2v; Nr. 5 (1523-1524), fol. 3r; Nr. 5 (15231524), fol. 3r; Nr. 6 (1524-1525), fol. 3v.

90 Eventuell die Ehefrau von Stephan Horenweil, der 1510 bis 1513 Kirchenmeister der Nikolaikirche war, vgl. StA Zwickau, Kaland 2, Nr. 1, fol. 17r; StA Zwickau, III Z4k, Nr. 71, Rechnungen Nikolaikirche, Nr. 10 (1510-1511), fol. 1v.

91 StA Zwickau, III Z Zk, Nr. 70, Rechnungen Marienkirche, Bd. 7: 1521-1534, Nr. 10 (1528-1530), fol. 4r-4v.

92 Anders als in Basel, vgl. Signori, Umstrittene Stühle (wie Anm. 85), S. 198.

93 StA Zwickau, III Z ${ }^{4 \mathrm{k}}$, Nr. 70, Rechnungen Marienkirche, Bd. 4: 1504-1508, Nr. 1 (1504-1506), fol. 1r; Fabian, Die Oswald Lasanschen Annalen (wie Anm. 31), S. 33.

94 Fabian, Die Oswald Lasanschen Annalen (wie Anm. 31), S. 4; Karant-Nunn, Zwickau in Transition (wie Anm. 8), S. 51 f. 
unter dem Vorwurf der Unterstützung der Zwickauer Propheten um Nicolaus Storch verhaftet wurde. ${ }^{95}$

\section{Seitenaltäre in der Marienkirche}

Eine weitere Möglichkeit der Gestaltung des Kirchenraumes durch die Gemeinde war die Stiftung von Seitenaltären und Privatkapellen, die im Spätmittelalter einen breiten Raum einnahm. ${ }^{96}$ Diese Altäre und Kapellen zergliederten zwar das Kirchenschiff in viele mehr oder minder private Bereiche, waren „Kirchen in der Kirche“, 97 boten jedoch auch vielfältige Identifikationsmöglichkeiten für die einzelnen Gemeindemitglieder. ${ }^{98}$

Im Allgemeinen entstanden Seitenaltäre in den Pfarrkirchen seit dem 6. Jahrhundert, in der Mehrzahl erst seit dem 13. Jahrhundert, allerdings konnten die ,Hochzeiten der Stiftungsbereitschaft ${ }^{`}$ in den einzelnen Pfarrkirchen stark variieren. ${ }^{99}$ In Lübeck war die ,stiftungsfreudigste' Zeit beispielsweise zwischen 1300 und 1349,100 während die Stiftungstätigkeit in Zwickau erst im späten 14. und dann vor allem im 15. Jahrhundert in Gang kam. In der Marienkirche gab es Anfang des 16. Jahrhunderts 23 Seitenaltäre mit 30 oder 31 Benefizien, in der Katharinenkirche zehn oder elf Altäre mit elf oder zwölf Benefizien, in der Nikolaikirche zwei Seitenaltäre mit zwei Benefizien und in der Spitalkirche St. Margaretha und Georg drei Seitenaltäre mit drei Benefizien. ${ }^{101}$ Die meisten Stiftungen in der Marienkirche wurden von Zwickauer Bürgern getätigt. Auffällig ist daneben die hohe Zahl von geistlichen Stiftern sowohl in der Marien- als auch in der Katharinenkirche. Allein in der Marienkirche stammen acht Altarstiftungen zum Teil oder auch ganz aus Stiftungen des Zwickauer Klerus. In der Katharinenkirche gingen die Altarstiftungen bis 1476 gänzlich auf die Initiative der Geistlichkeit zurück. Bei der Stiftung des Frühmessaltars Heiligkreuz, des ersten Seitenaltars in der Marienkirche (1291), beispielsweise war neben dem Kloster Eisenberg auch Heinrich von Salburg, der Bruder des ersten bekannten Zwickauer Bürgermeisters

95 Karant-Nunn, Zwickau in Transition (wie Anm. 8), S. 113 f.

96 Vgl. Hans Erich Feine, Kirchliche Rechtsgeschichte. Die katholische Kirche, Köln/ Graz ${ }^{4}$ 1964, S. 421 f.; Hans Liermann, Geschichte des Stiftungsrechts, ergänzt durch ein Geleitwort von Axel Freiherr von Campenhausen und eine Abhandlung von Michael Borgolte, Tübingen 22002, S. 111 f.; Justin E. A. Kroesen, Seitenaltäre in mittelalterlichen Kirchen. Standort - Raum - Liturgie, Regensburg 2010, der sich mit der Auswirkung der mittelalterlichen Seitenaltäre auf das Raumerlebnis und die Wahrnehmung der Liturgie beschäftigte.

97 Boockmann, Bürgerkirchen (wie Anm. 3), S. 15.

98 Kroesen, Seitenaltäre (wie Anm. 96), S. 11.

99 Sabine Graf, Das Niederkirchenwesen der Reichsstadt Goslar im Mittelalter, Hannover 1998, S. 169; Kroesen, Seitenaltäre (wie Anm. 96), S. 10.

100 Wolfgang Prange, Vikarien und Vikare in Lübeck bis zur Reformation (Veröffentlichungen zur Geschichte der Hansestadt Lübeck B/40), Lübeck 2003, S. 18 f.

101 Vgl. dazu Kahleyss, Zwickau (wie Anm. 2), S. 383. 
Conrad von Salburg, ${ }^{102}$ beteiligt. ${ }^{103}$ Die nächste Stiftung eines Seitenaltares in der Marienkirche, des Altars St. Kunigunde, tätigte die größte und älteste Zwickauer Bruderschaft, die Kalandbruderschaft. Das Stiftungsdatum lässt sich auf die Zeit kurz vor 1365 datieren. ${ }^{104}$ Die folgenden Stiftungen der Altäre St. Martin, St. Felix und Adauctus (1385) und St. Levin wurden in der Mehrzahl von Zwickauer Ratsfamilien, teilweise auch von den Bürgermeistern selbst ins Leben gerufen. ${ }^{105}$

Der bereits erwähnte Altar St. Felix und Adauctus beispielsweise wurde 1385 von dem Zwickauer Bürger Hans Kirtzscher (Kürtzscher) und dessen Sohn Franz gestiftet. 106 Der Entschluss zur Stiftung dieses Altars ist wahrscheinlich dem Vater Hans zuzuschreiben, die Realisierung fiel seinem Sohn Franz zu. Dieser trat nicht nur in der Marienkirche als Stifter auf, sondern verwendete auch eine hohe Summe für die Stiftung eines Kaplanats in der Spitalkirche St. Spiritus. Anders als sein Vater, der sich in keinem hohen Amt nachweisen lässt, war Franz Kirtzscher nicht nur Ratsherr 1402, sondern 1406, 1408 und 1410 auch Bürgermeister in Zwickau. ${ }^{107}$ Beide stellen den von Wolfgang Schmid beschriebenen Typus der „Neubürger",108 oder in diesem Fall der „Aufsteiger“, dar, der versuchte, damit seine neu erworbene soziale oder politische Stellung zu dokumentieren und meist größere Summen stiftete. ${ }^{109}$

Ein frühes Beispiel der im Spätmittelalter stark verbreiteten Bruderschaftsaltäre ${ }^{110}$ ist der Fronleichnamsaltar, der 1406 von der gleichnamigen Bruderschaft

102 Conrad von Salburg war von 1297 bis 1307/08 Bürgermeister in Zwickau; vgl. Hans PATZE (Hg.), Altenburger Urkundenbuch, Bd. 1: 976-1350 (Veröffentlichungen der Thüringischen Historischen Kommission 5), Jena 1955, S. 330 f., Nr. 409; StA Zwickau, A*A III 19, Nr. 1; Emil Herzog, Chronik der Kreisstadt Zwickau, Zweiter Teil, Zwickau 1845 (Reprint Stuttgart 1999), S. 874, Anhang Nr. 7; CDS II/6: Urkundenbuch der Stadt Chemnitz und ihrer Klöster, hrsg. von Hubert Ermisch, Leipzig 1879, S. 7, Nr. 9.

103 StA Zwickau, III x ${ }^{1}$, 141b (Zwickauer Rechtsbuch von 1348), fol. 5v-6r; CDS II/21: Urkundenbuch der Stadt Zwickau, Teil 1: Die urkundliche Überlieferung 1118-1485, Bd. 1: 1118-1399, bearb. von Henning Steinführer, Hannover 2014, S. 23, Nr. 26; Kahleyss, Zwickau (wie Anm. 2), S. 74.

104 StA Zwickau, Kaland 1, Nr. 6, fol. 2v; CDS II/2 I: S. 127, Nr. 145; Emil Herzog, Die Zwickauer Kalandbrüderschaft, in: Zwickauer Wochenblatt (1864), Nr. 247 f., S. 16011603.

105 Vgl. Kahleyss, Zwickau (wie Anm. 2), S. 390.

106 ThHStA Weimar, EGA, Reg. O 156, fol. 213r; StA Zwickau, Kopialbuch III, fol. 5-7; Kaland 1, Nr. 5; Kaland 5, Nr. 2; A*A I 25, Nr. 1: Ratsbestätigungen 1387-1460, Eintrag von 1402; CDS II/2 I: S. 160, Nr. 185.

107 StA Zwickau, A*A I 25, Nr. 1: Ratsbestätigungen 1387-1460, Eintrag von 1408.

108 Wolfgang Schmid, Stifter und Auftraggeber im spätmittelalterlichen Köln, Diss. phil. Trier (Veröffentlichungen des Kölnischen Stadtmuseums 11), Köln 1990, S. 506 f. Heinrich Dormeier stellte hingegen fest, dass sehr viele Lübecker ,Neubürger ${ }^{\varsigma}$ zwar großzügige Schenkungen für kirchliche Einrichtungen testierten, aber meist in erster Linie die Kirchen ihres Heimatortes bedachten, vgl. Heinrich Dormeier, Das laikale Stiftungswesen in spätmittelalterlichen Pfarrkirchen, in: Bünz/Fouquet, Die Pfarrei im späten Mittelalter (wie Anm. 23), S. 279-340, hier S. 287.

109 Schmid, Stifter und Auftraggeber (wie Anm. 108), S. 506 f.

110 Kroesen, Seitenaltäre (wie Anm. 96), S. 11. 
in der Marienkirche gestiftet wurde. ${ }^{111}$ Von den fünf namentlich genannten Mitgliedern ${ }^{112}$ lassen sich zwei, Johannes Horlemann und Nicolaus Lazan, im Rat nachweisen, während einer wahrscheinlich mit Conrad Holtzborn, der 1401 Bürgermeister war, verwandt war. ${ }^{113}$ Ein vierter, Petrus Gerhard, könnte identisch mit dem 1387 erwähnten gleichnamigen Schöffen sein. ${ }^{114}$ Danach stockte die Stiftungsbereitschaft in der Marienkirche aus ungeklärten Gründen für ca. zwei Jahrzehnte, setzte danach jedoch verstärkt wieder ein. Möglicherweise könnte dies in einen Zusammenhang zu den Hussiteneinfällen zu setzen sein, die Zwickau zwar erst 1430 unmittelbar bedrohten,115 jedoch schon vorher einen Rückgang der Handelstätigkeit und damit wirtschaftliche Schwierigkeiten mit sich brachten. ${ }^{116}$ Ende des 15. Jahrhunderts machte sich schließlich der Erfolg einiger Zwickauer im Schneeberger Bergbau bemerkbar. Dazu gehörte Martin Römer, der mit seinen Stiftungen auch neue Akzente setzte, was sich beispielsweise an der Einrichtung des Reichen Almosens, einer süddeutschen Entwicklung, zeigte. In den ersten Jahren des 16. Jahrhunderts folgte die Stiftung eines Benefiziums des, Gestifts Exulum animarum' der Elendenbruderschaft in Zwickau, ${ }^{117}$ allerdings wahrscheinlich ohne die Errichtung eines Altares.

Im Unterschied zur Marienkirche nahm die Stiftungstätigkeit in der Katharinenkirche erst Ende des 15. Jahrhunderts zu. Die drei ersten Altarstiftungen St. Maria Magdalena (1332), St. Barbara (vor 1378) und Decem milium militum (1445) in der Katharinenkirche gingen größtenteils auf geistliche Stifter zurück. ${ }^{118}$ Erst in den siebziger Jahren des 15. Jahrhunderts erlebte die Katharinenkirche ein wahres ,Stiftungsfieber‘. Allein im Jahr 1479 wurden drei Seitenaltäre, Trium regum und St. Barbara, Conceptio Mariae und Commemoratio Mariae, durch den Zwickauer Bürger Caspar Richter und seine Frau Barbara gestiftet. ${ }^{119}$ Richters Stiftung umfasste jedoch nicht nur ein Seelgerät, sondern er finanzierte mit der Einrichtung des Altarlehens Trium regum und St. Barbara eine feste Predigerpfründe an der Katharinenkirche.

111 StA Zwickau, A*A Kopialbuch II, fol. 13 f.; Kopialbuch III, fol. 9v-11.

$112 \mathrm{Vgl}$. StA Zwickau, A*A III 5, Nr. 11.

113 StA Zwickau, A*A I 25, Nr. 1: Ratsbestätigungen 1387-1460, Eintrag von 1401, 1405, 1408.

114 StA Zwickau, A*A III 5, Nr. 6.

115 Curt Vogel, Zwickau im Hussitenkriege, in: Alt-Zwickau (1924), Nr. 4, S. 13-16.

116 Max Mueller, Das Tuchmacher-Handwerk und der Tuchhandel in Zwickau in Sachsen. Ein Beitrag zur Wirtschafts-Geschichte Sachsens, Diss. phil. Leipzig, Ohlau 1929, S. 95 .

117 StA Zwickau, Kaland 35, Nr. 4, fol. 4r; Herzog, Chronik II (wie Anm. 102), S. 229, 842, 861; Ders., Chronik I (wie Anm. 35), S. 95.

118 Vgl. CDS II/2 I, S. 60, Nr. 78, S. 167, Nr. 147; CDS II/20: Urkundenbuch der Stadt Zwickau, Teil 2: Das älteste Stadtbuch 1375-1481, bearb. von Jens Kunze, Hannover 2012, Nr. 779; Kahleyss, Zwickau (wie Anm. 2), S. 389.

119 StA Zwickau, Kaland 6, Nr. 6; A*A III 24, Nr. 9; Kaland 7; A*A III 28, Nr. 10; KAHLEYss, Zwickau (wie Anm. 2), S. 397. 
Auch der Bau der einzigen Familienkapelle Zwickaus, der Römerkapelle, lässt sich in den Kontext der Zwickauer Stiftertradition einordnen. Im Allgemeinen gelten der Einbau und der damit verbundene hohe finanzielle Aufwand, den einzelne Gemeindemitglieder leisteten, als Kennzeichen „reicher, unabhängiger Bürgerstädte“. ${ }^{120}$ Der kapitalintensive ${ }^{121}$ Bau von Privat- und Familienkapellen begann dementsprechend in großen Städten wie Florenz oder Köln bereits im 12. und 13. Jahrhundert. ${ }^{122}$ In den norddeutschen Hansestädten lässt sich die ,Hochphase' des Kapellenbaus um 1380 beginnend bis zur Reformation ansetzen, ${ }^{123}$ während sich im süddeutschen Raum der Bau von Einsatzkapellen in den Pfarrkirchen erst Anfang des 15. Jahrhunderts nachweisen lässt. ${ }^{124}$ Die Besitzer der Privatkapellen waren meist Ratsherren. ${ }^{125}$ In Zwickau konnte sich der Bau von Privatkapellen zunächst nicht durchsetzen. Der Bauplan des 1506 begonnenen Langhauses der Marienkirche beinhaltete zwar den Einbau von Einsatzkapellen, die zwischen den eingezogenen Strebepfeilern und unter den das Kirchenschiff auf drei Seiten umspannenden Emporen lagen, ${ }^{126}$ eine explizite Nutzung dieser ,Nischen“ als Privatkapellen ist jedoch nicht belegbar. Die als Grablege der Familie Römer erbaute Kreuzkapelle wurde bereits 1479 an das westliche Langhausjoch als Kapellenanbau angesetzt. ${ }^{127}$ Die Kapelle war durch ein Gitter vom übrigen Kirchenraum abgetrennt und so für die Allgemeinheit nicht zugänglich. Martin Römer sorgte für die reiche Ausstattung der Kapelle: So stiftete er 1479160 Gulden und ein goldenes Kreuz mit fünf mit Saphiren besetzten „Splittern vom Kreuz Christi“ für diese Kapelle. ${ }^{128}$ Diese sollen laut dem Bericht des Chronisten Peter

120 Antje Heling, Die Nutzung der Chorkapellen in norddeutschen Pfarrkirchen, in: Anna Morath-Fromm (Hg.), Kunst und Liturgie. Choranlagen des Spätmittelalters ihre Architektur und Nutzung, Ostfildern 2003, S. 141-157, hier S. 141.

121 Weitzel, Stralsund (wie Anm. 82), S. 104.

122 Annegret Höger, Studien zur Entstehung der Familienkapelle und zu Familienkapellen und -altären des Trecento in Florentiner Kirchen, Diss. phil. Bonn 1976, S. 62 f.; Kroesen, Seitenaltäre (wie Anm. 96), S. 53.

123 Heling, Chorkapellen (wie Anm. 120), S. 141.

124 Antje Grewolls, Die Kapellen der norddeutschen Kirchen im Mittelalter. Architektur und Funktion, Kiel 1999, S. 45.

125 Ebd., S. 127.

126 Vgl. beispielsweise Georg Buchwald (Hg.), Neue Sächsische Kirchengalerie. Ephorie Zwickau, Leipzig 1902, Sp. 75. Emporen besaß die Marienkirche allerdings mindestens seit 1443, vgl. WeIssbach, Marienkirche (wie Anm. 7), S. 45.

127 Grewolls, Die Kapellen der norddeutschen Kirchen (wie Anm. 124), S. 19; KüHN, Baugeschichte (wie Anm. 5), S. 9.

128 Otтo Clemen, Reformationsgeschichtliches aus dem Zwickauer Ratsarchiv, Teil V: Martin Römers goldenes Kreuz in der Marienkirche zu Zwickau, in: Archiv für Reformationsgeschichte 26 (1929), S. 188-203, wiederabgedruckt in: OTTO ClEMEN, Kleine Schriften zur Reformationsgeschichte (1897-1944), Band V: 1922-1932, hrsg. von Ernst Koch, Leipzig 1984, S. 426 f.; Hildebrand, St. Mariä (wie Anm. 32), S. 51; Fabian, Die Oswald Lasanschen Annalen (wie Anm. 31), S. 27; Emil Herzog, Martin Römer. Ein biographischer Beitrag zur sächsischen Culturgeschichte, in: Mittheilungen des Königlich Sächsischen Vereins für Erforschung und Erhaltung vaterländischer Geschichts- und Kunstdenkmale 14 (1865), S. 49-63, hier S. 57 f. 
Schumann über Österreich aus Konstantinopel nach Zwickau gekommen sein, wo sie der Salzburger Bürger Hans Schwieger dem Amtshauptmann Martin Römer vorlegte. ${ }^{129}$ Römer kaufte diese Reliquie für den Preis von 100 Mark Silber und einem Kux in der Müntzerzeche auf dem Schneeberg, der zu dieser Zeit 1000 Gulden wert gewesen sein soll. In der Kapelle fanden Martin Römer, sein Bruder Nicolaus und Hans Römer ihre letzte Ruhestätte. ${ }^{130}$ Hildebrand war der Ansicht, dass es sich bei dem Letztgenannten um Martin Römers Vater handele. Jedoch schloss Herzog aus der in der Kapelle angebrachten Tafel mit der Aufschrift Die Römer Gebrüdere als Martin, Niclas und Hans ${ }^{131}$ seien dort begraben, es handele sich um den Stiefbruder Martin Römers. ${ }^{132} 1484$ stifteten Nicolaus Römer, Katharina von Hermannsgrün (Martin Römers Witwe), der Bergschreiber Hieronymus Beyer (Martin Römers Schwager) und Caspar von Sala 600 Gulden mit 24 Gulden jährlichen Zinsen für eine ewige Kreuzmesse, die jeden Freitag zelebriert werden sollte. ${ }^{133}$

Die Reformation brachte durch die Abschaffung der Privatmessen eine grundlegende Änderung des Kirchenraumes mit sich. In Zwickau erfolgte bereits 1523 die Einrichtung eines Gemeinen Kastens, ${ }^{134}$ und alle Bruderschaften wurden bis auf den Kaland aufgelöst (dieser folgte 1527). ${ }^{135}$ Ostern 1525 wurden alle altgläubigen Bräuche abgeschafft. ${ }^{136}$ Laut Hildebrand wurden zu diesem Zeitpunkt alle Seitenaltäre abgebrochen. ${ }^{137}$ In den meisten Fällen ist das weitere Schicksal der Zwickauer Altäre nicht geklärt, allerdings legt das der bekannten nahe, dass es noch einige Zeit dauerte, bis sie aus dem Kirchenschiff entfernt waren. So beschwerte sich beispielsweise der 1554 zum Pfarrer berufene Johann Petrejus über die katholischen Kunstwerke in der Marienkirche, insbesondere über den Hochaltar von Michael Wolgemut, den er mit einem neuen Motiv versehen lassen wollte. Der Rat wies dies jedoch mit Verweis auf fehlende finanzielle Mittel ab. ${ }^{138}$ Ein anderes heute in der Katharinenkirche aufgestelltes Retabel stammt ursprünglich von dem bereits erwähnten Kunigundenaltar der Zwickauer Kalandbruder-

129 Schumann, Annalen (wie Anm. 31), fol. 428v-431v.

130 Toвias Schmidt, Chronica Cycnea oder Beschreibung der sehr alten / Loblichen / und Churfürstlichen Stadt Zwickau [...], Zwickau 1656 (Ratsschulbibliothek Zwickau, 66.7.14/1), S. 59.

131 Ebd.

132 Herzog, Martin Römer (wie Anm. 128), S. 58.

133 StA Zwickau, A*A III 24, Nr. 16; A*A III 24, Nr. 17; A*A III 24, Nr. 19.

134 Helmut Bräuer/Elke Schlenkrich, Kampf um den Gemeinen Kasten in Zwickau. Städtische Armenversorgung in der Auseinandersetzung zwischen Ratstisch, Gasse und Kanzel, in: Herbergen der Christenheit 28/29 (2004/2005), S. 57-88.

135 StA Zwickau, Kaland 2, Nr. 1.

136 Anne-Rose FröHlich, Die Einführung der Reformation in Zwickau, in: Mitteilungen des Altertumsvereins für Zwickau und Umgegend 12 (1919), S. 1-74, hier S. 33; Schumann, Annalen (wie Anm. 31), fol. 200v.

137 Hildebrand, St. Marï̈ (wie Anm. 32), S. 49.

138 Otto Langer, Der Kampf des Pfarrers Joh. Petrejus gegen den Wohlgemuthschen Altar in der Marienkirche, in: Mitteilungen des Altertumsvereins für Zwickau und Umgegend 11 (1914), S. 31-49. 
schaft in der Marienkirche und gelangte vermutlich über den Umweg der abgerissenen Kirche des Franziskanerklosters an seinen heutigen Standort. ${ }^{139}$

$$
* * *
$$

Noch heute rufen der Bau und die vorhandenen Reste der mittelalterlichen Ausstattung der Marienkirche, des heutigen Doms St. Marien, Bewunderung hervor und dokumentieren die Ambitionen des Zwickauer Rates und der Gemeinde beim Bau des Kirchengebäudes und die Identifikation mit ihrer Pfarrkirche. Im vorliegenden Beitrag wurde ein Versuch unternommen, die Motivationen für den Beginn, die Weiterführung und die Ausstattung dieses spätmittelalterlichen Bauvorhabens zu beleuchten. Unter anderem gehörten dazu die Konkurrenz des Rates zum Kloster Eisenberg, welches das Patronat über die Zwickauer Kirchen innehatte, der überregionale Vergleich mit den Kirchen anderer Städte wie Nürnberg und Schneeberg, bedeutende wirtschaftliche Möglichkeiten durch Erfolge im Bergbau und eine große Stiftungsbereitschaft seitens der Pfarrgemeinde. Auch Veränderungen in der Liturgie, bedingt durch die Einführung der Reformation, wirkten sich gravierend auf die Gestaltung des Kirchenraumes aus, was sich beispielsweise an den Seitenaltären zeigt, die vorher das Langhaus dominierten. Dazu gehört auch der Einbau von Kirchenstühlen, welche die Nutzung und die Sichtachsen des Kirchenraumes erheblich veränderten. Dies alles ist als Einblick in die Motivlage der beteiligten gesellschaftlichen Gruppen zu verstehen, ohne Anspruch auf Vollständigkeit. Gänzlich separieren lassen sich die einzelnen Faktoren ohnehin nicht, bildeten jedoch in ihrer Gesamtheit den Hintergrund für die jahrzehntelangen Umbauarbeiten am Gebäude der Marienkirche.

139 Zuletzt dazu Michael KüHN, Wie das von Lucas Kranach gemalte Altarretabel in die St. Katharinenkirche gekommen ist, in: Informationshefte Dom St. Marien 14 (2010), S. 17; vgl. zukünftig die Beiträge in: Thomas Pöpper (Hg.), Das Cranach-Retabel in der Zwickauer St. Katharinenkirche. Deutung, Dokumente und Restaurierung, erscheint voraussichtlich 2017, darin: Julia KaHLEYss, Der Zwickauer Kaland. 